J. ZHENG

KODAI MATH. J.

10 (1987), 328-334

\title{
ON THE FACTORIZATION OF SEVERAL CLASSES OF ENTIRE FUNCTIONS
}

\author{
BY JIANHUA ZHENG
}

\section{Introduction and statement of results.}

Gross 1 discussed the factorization of the function in the form like $F(z)=$ $H(z)+\exp (\lambda z+G(z))$, where both $H(z)$ and $G(z)$ are periodic entire functions with period $\tau$. But the present author found that Theorem 7 of Gross 1 seem to be wrong. In his Theorem 7 , Gross showed that $F(z)$ is prime, if $\lambda \tau /(\pi i)$ is an irrational. However, $[H(w)+\exp (\lambda w+G(w))] \circ(z+H(z))$ can be expressed in the form of $F(z)$ too.

In this paper, the present author is to correcte the mistake of Theorem 7 of Gross 1 and to consider the factorization of the entire functions of the other class to get some interesting, periodic, prime entire functions. We assume that the reader is familiar with the concepts of the factorizations of entire functions.

THEOREM 1. Let $F(z)$ be $H(z)+\exp (\lambda z+G(z))$, where both $H(z)$ and $G(z)$ are periodic entire functions with period $\tau$ and $\lambda \tau /(\pi i)$ is an irrational. If $F(z)=f(g)$, where both $f$ and $g$ are non-linear entire functions, then we have the forms of $f$ and $g$, respectively,

$$
f(w)=H_{1}(w)+\exp \left(c w+H_{2}(w)\right), \quad g(z)=d z+H_{3}(z),
$$

where $d c=\lambda$, both $H_{1}$ and $H_{2}$ are periodic functions with period $d \tau$ and $H_{3}$ is periodic with period $\tau$.

THEOREM 2. Let $F(z)=H(z)+\exp (\lambda z+G(z))$, where $H$ and $G, \lambda$ are as in Theorem 1 and $H(z) \neq$ constant. $F(z)$ is prime, if one of the following condition holds:

(i) There do not exist two non-constant periodic entire functions $H_{1}(z)$ and $H_{2}(z)$ with period $\tau$ such that $H(z)=H_{1}\left(z+H_{2}\right)$;

(ii) For any $\varepsilon>0$, there are two unbounded sequence $\left\{r_{k}\right\}$ and $\left\{r_{k}^{\prime}\right\}$ of positive real number such that $M\left(r_{k}, G\right)<e_{2}\left(\varepsilon r_{k}\right)$ and $M\left(r_{k}^{\prime}, H\right)<\exp \left(\varepsilon M\left(r_{k}^{\prime} / 2, G\right)\right)$, where $e_{2}(z)=\exp (\exp z)$;

(iii) $G(z)=$ constant .

Received April 10, 1987. 
THEOREM 3. Let $F(z)=H(z) \cdot \exp (G(z)+S(z))$, where $G(\neq$ constant $)$ and $H$ are as in Theorem 1 with $\rho(G)<+\infty$ and $\rho(H)<+\infty(\rho(H)$ denotes the order of $H)$ and $S$ is a polynomial. And let $H$ have an infinite number of zeros which all lie on a straight line. Then the factorization of $F(z)$ only can be one of the following forms:

(i) $F(z)=f(P(z))$, where $P$ is a polynomial of degree 2 and $f$ an entire function;

(ii) $F(z)=g(z)^{n}$, where $g$ is an entire function and $n$ an integer greater than 1 ;

(iii) $H(z)=Q(g(z)) e^{-S(z)}, \quad G(z)=U(g(z))$, and $S(z)=(2 n \pi i / m \tau) z+c$, where $m(>0)$ and $n$ are integers, $Q$ is a rational function and $U$ an entire function of order zero, $g$ is periodic with period $m \tau$.

By Theorem 3, we have immediately

CoROllaRY 1. Let $F(z)$ be $H(z) e^{G(z)}$, where both $H$ and $G$ are as in Theorem 3. $F(z)$ is prime, if the following conditions hold:

(i) $H(z)$ has at least one simple zero;

(ii) For any complex number b, either $H(z+b)$ or $G(z+b)$ is not even;

(iii) $H(z)$ and $G(z)$ have no common periodic right factors.

Further we have

COROLlaRY 2. For any non-negative integers $k$ and $n, F(z)=\cos ^{n} z \cdot \sin z$ $\cdot \exp \left(\cos ^{2 k+1} z\right)$ is prime.

Remark. (a) By Pólya's lemma (cf. Pólya 5) and the facts that the lower order $\lambda(G)$ of any periodic entire function $G$ is greater than 1 or equal to 1 and $\sigma(G)>0\left(\sigma(G)=\liminf _{r \rightarrow+\infty} T(r, G) / r^{\lambda(G)}\right)$ (cf. Gross 1) and that for any nonperiodic entire function, the prime factorization in the sense of entire function is equivalent to the prime factorization in the sense of generality (cf. Gross 6), we can easily verify Theorem 2 , so we omit the proof of Theorem 2 .

(b) From (iii) of Theorem 2, we know that the entire functions $e^{\lambda_{1} z}+e^{\lambda_{2} z}$ and $H\left(e^{z}\right)+e^{\lambda z}$ are prime, where both $\lambda_{1} / \lambda_{2}$ and $\lambda$ are irrationals, and $H(w)$ is a holomorphic function in $0<|w|<+\infty$. Evidently, their derived functions of any order are also prime.

(c) Recently, G.D. Song and J. Huang 7 showed that $\sin z \cdot \exp (\cos z)$ is prime. However, by Corollary 1 and the fact that entire functions $F$ and $F^{\prime}$ have no common right factor $g$ unless $g=a \cdot \exp (b z+c)+d$, where all $a, b, c$ and $d$ are constants (cf. Gross $8, \mathrm{p} 121$ ), we easily get more general prime functions than theirs. In fact, we easily verify that both $\cos ^{n} z \cdot \sin z \cdot \exp \left(\cos ^{n+1} z\right)$ and $\sin ^{n} z$ $\cdot \cos z \cdot \exp \left(\sin ^{n+1} z\right)$ are prime, for any integer $n \geqq 0$. Evidently, the derived function and the second power of these functions are not pseudo prime. It follows that product and derivative of the prime functions are not always pseudo prime.

In order to prove our results, we need some lemmas. 
LEMMA 1. Let $F(z)=H(z)+\exp (\lambda z+G(z))=f(g)$, where $H$ and $G, f$ and $g, \lambda$ are as in Theorem 1. If $g(z)=T_{1}(z)+z \cdot \exp \left(k z+T_{2}(z)\right)$, where $T_{\imath}(i=1,2)$ are periodic with period $\tau$ and $e^{\tau k}=1$, then $\exp \left(k z+T_{2}(z)\right)=$ constant.

By making a little modification of the method of Urabe 2, we can verify Lemma 1.

LEMMA 2. Let $F(z)=H(z)+Q(z)$, where $H(z)$ ( $\neq$ constant) is an entrre function of finite lower order, periodic with period $\tau$ (say) and $Q(z)(\neq$ constant) a polynomial. Then $F(z)$ is left-prime in the sense of entire function. (cf. Urabe 3).

LEMMA 3 (cf. Edrei 4). Let $f(z)$ be an entire function. Assume that there exist an unbounded sequence $\left\{a_{n}\right\}$ of fint complex number such that all the roots of $f(z)=a_{n}(n=1,2, \cdots)$ lie on a straight line. Then $f(z)$ is a polynomial of degree at most 2 .

\section{Proof of Theorems.}

Proof of Theorem 1. Assume $\tau=1$ without the loss of generality. Then we have

$$
f(g(z+1))-f(g(z))=\left(e^{\lambda}-1\right) \exp (\lambda z+G(z)) \neq 0 .
$$

It shows that

$$
g(z+1)-g(z)=\exp \left(V_{1}(z)\right),
$$

where $V_{1}$ is an entire function. Further, we have

$$
g(z+2)-g(z+1)=\exp \left(V_{1}(z+1)\right) .
$$

By the same method as in the above, we can find out an entire function $V_{2}(z)$ such that

$$
g(z+2)-g(z)=\exp \left(V_{2}(z)\right) .
$$

Combining (2), (3) and (4), we get immediately

$$
\exp \left(V_{1}(z)\right)+\exp \left(V_{1}(z+1)\right)=\exp \left(V_{2}(z)\right) .
$$

Then if follows by Borel's Theorem (cf. Gross 8, p 165) and (5) that $V_{1}(z+1)=$ $V_{1}(z)+k$, where $k$ is a constant. Put $G_{1}(z)=V_{1}(z)-k z$. Then $G_{1}(z)$ is periodic with period 1. And it follows from (2) that

$$
g(z+1)=g(z)+\exp \left(k z+G_{1}(z)\right) .
$$

We consider two cases of the following, separately.

(I) The case when $e^{k} \neq 1$. We obtain from (6) without difficulty 


$$
g(z)=H_{1}(z)+\left(e^{k}-1\right)^{-1} \cdot \exp \left(k z+G_{1}(z)\right),
$$

where $H_{1}$ is periodic with period 1 . Analysing the proof of Theorem 7 of Gross 1, we know that the expression (7) of $g$ does not hold.

(II) The case when $e^{k}=1$. We easily get from (6) the form of $g(z)$

$$
g(z)=H_{1}(z)+z \cdot \exp \left(k z+G_{1}(z)\right),
$$

where $H_{1}$ is as in the above. Thus it follows by Lemma 1 and (8) that $g(z)$ can be written as $g(z)=H_{1}(z)+d z$, for some non-zero complex number $d$. Since $F(z)=f\left(H_{1}(z)+d z\right)$, we have immediately

$$
f\left(H_{1}(z)+d z+d\right)-f\left(H_{1}(z)+d z\right)=\left(e^{\lambda}-1\right) \cdot \exp (\lambda z+G(z)) \neq 0 .
$$

Function $H_{1}(z)+d z$ can assume any complex number, hence it shows from (9) that

$$
f(w+d)-f(w)=\exp (V(w)),
$$

for some entire function $V(w)$. From (9) and (10), we have $\exp \left(V\left(d z+H_{1}(z)\right)\right.$ $=\left(e^{\lambda}-1\right) \cdot \exp (\lambda z+G(z))$, i. e. $V\left(d z+H_{1}(z)\right)=\lambda z+G(z)+c_{1}$, for some complex number $c_{1}$. Differentiating both the sides of the former equality, we have $V^{\prime}\left(H_{1}(z)+d z\right) \cdot\left(d+H_{1}^{\prime}(z)\right)=\lambda+G^{\prime}(z)$. It follows that $V^{\prime}\left(H_{1}(z)+d z\right)$ is periodic with period 1 . Hence we have

$$
\begin{gathered}
V^{\prime}\left(H_{1}(z)+d z+d\right)=V^{\prime}\left(H_{1}(z)+d z\right), \\
V^{\prime}(w+d)=V^{\prime}(w), \quad V(w+d)=V(w)+c, \quad(c: \text { a constant }) .
\end{gathered}
$$

Put $G_{2}(w)=V(w)-(c / d) w$. Then $G_{2}$ is periodic with period $d$. Thus it follows from (10) that

$$
f(w+d)-f(w)=\exp \left(\frac{c}{d} \cdot w+G_{2}(w)\right)
$$

If $e^{c}=1$, we can easily find out a periodic entire function $H_{2}(w)$ of period $d$ such that $f(w)$ has the form $f(w)=H_{2}(w)+(w / d) \cdot \exp \left((c / d) w+G_{2}(w)\right)$. From the forms of $F(z)$ and $g(z)$, we know that this expression of $f(w)$ contradict $F(z)=f(g)$. Therefore $e^{c} \neq 1$. Thus it follows from (11) that $f(w)$ can be expressed as

$$
f(w)=H_{2}(w)+\exp \left(\frac{c}{d} w+G_{2}(w)\right)
$$

where $\mathrm{H}_{2}$ is periodic with period $d$. Comparing the forms of $F$ and $f(g)$, we have $c=\lambda$. Thus we complete the proof of Theorem 1 .

Proof of Theorem 3. Let $F(z)$ be $f(g)$. We divide four cases of the following to be considered, separately.

(I) The case when $g$ is a polynomial. Then $f$ certainly is transcendental 
entire. $F(z)$ has infinite many zeros, so does $f(z)$. By Lemma $3, g$ must be of degree at most 2.

(II) The case when $g$ is a transcendental entire function and $f$ is entire. By Lemma $3, f(w)$ only has a finite number of zeros. Hence $f(w)$ can be written as $f(w)=Q(w) e^{U(w)}$ with a polynomial $Q$ and an entire function $U$. We treat two subcases of the following, separately.

(II. 1) The subcase when $Q$ has two distinct zeros. Then $g$ is of finite order. By $\rho(H)<+\infty$ and $F=f(g)$, we have

$$
H(z)=Q(g(z)) \cdot \exp (M(z)),
$$

where $M$ is a polynomial. Further, we easily get

$$
U(g(z))=G(z)+S(z)+M(z)+2 k_{0} \pi i,
$$

for some integer $k_{0}$.

If $S(z)+M(z) \neq$ constant, it follows by Lemma 2 and (13) that $U$ is linear. Put $U(w)=a w+b,(a \neq 0)$. Therefore we have the equality $g(z)=1 / a(G(z)+S(z)$ $\left.+M(z)+2 k_{0} \pi i-b\right)$. Taking a point $z_{0}$ such that $H\left(z_{0}\right) \neq 0$, we establish from (12) the equality

$$
H\left(z_{0}\right)=Q\left(\frac{1}{a} G\left(z_{0}\right)+\frac{1}{a}\left(S\left(z_{0}+n \tau\right)+M\left(z_{0}+n \tau\right)+2 k_{0} \pi i-b\right)\right) \cdot \exp \left(M\left(z_{0}+n \tau\right)\right),
$$

for any positive integer $n$. However, the right side of (14) approaches 0 or $\infty$ as $n \rightarrow+\infty$. This is absurd. Hence $S(z)+M(z)=$ constant. It follows from (13) that $U$ certainly is of order zero (cf. Polya 5) and $g$ a periodic function of period $m \tau$ for some positive number $m$ (cf. Gross $8, \mathrm{p} \mathrm{106).} \mathrm{Thus} \mathrm{from} \mathrm{(12),}$ $\exp (M(z))$ is periodic with period $m \tau$, so is $\exp (S(z))$. Then $S(z)$ must have the form $S(z)=(2 n \pi i / m \tau) z+c$ with an integer $n$ and a constant $c$. Thus we obtain the factorization of case (iii).

(II.2) The subcase when $Q(w)=u\left(w-w_{0}\right)^{n}$, where $u$ is a constant and $n$ a positive integer. Then $f(w)$ can be written as

$$
f(w)=u\left(w-w_{0}\right)^{n} \cdot \exp (U(w)) .
$$

We may assume that $U(w) \neq$ constant. In fact, if $U(w)=$ constant, we get the factorization of case (ii). Further, $g(z)$ can be written as

$$
g(z)=w_{0}+h(z) \cdot \exp (W(z)),
$$

where $W(z)$ and $h(z)$ are both entire functions and $\rho(h)<+\infty$. Thus it follows that

$$
H(z)=h(z)^{n} \cdot \exp (N(z)),
$$

for some polynomial $N(z)$. Furthermore, we have

$$
U(g(z))=G(z)+S(z)+N(z)-n W(z)+2 k_{0} \pi i,
$$


for some integer $k_{0}$. Since $\rho(G)<+\infty$, it follows from (16) and (18) that $W$ is a polynomial. According to the same method as in subcase (II.1), we can show

$$
S(z)+N(z)=n W(z)+c_{1}, \quad\left(c_{1}: \text { a constant }\right) .
$$

Thus it follows from (16) and (19) that $\left(g-w_{0}\right)^{n}=h^{n} e^{n W}=H \exp \left(S-c_{1}\right)$, i. e.

$$
H(z)=\left(g(z)-w_{0}\right)^{n} \cdot \exp \left(c_{1}-S(z)\right) .
$$

From (18) and (19), we have immediately the equality $G(z)=U(g(z))-\left(2 k_{0} \pi i+c_{1}\right)$. Then $U$ is an entire function of order zero and $g$ is periodic with period $m \tau$ for some positive integer $m$. From (20), we can write $S(z)$ as $(2 s \pi i / m \tau) \cdot z+c$ with a constant $c$ and an integer $s$. Then we get the factorization of case (iii).

(III) The case when $g$ is transcendental entire and $f$ non-entire meromorphic. Then we can write $f(w)$ and $g(z)$ as $f^{*}(w) /\left(w-w_{1}\right)^{q}$ and $w_{1}+$ $\exp (W(z))$, respectively, where $q$ is a positive integer and $w_{1}$ a complex number, $f^{*}$ and $W$ are both entire functions and $f^{*}\left(w_{1}\right) \neq 0$. By Lemma $3, f^{*}$ only has finite many zeros. Then we can write $f^{*}(w)$ as $Q(w) \exp (U(w))$, where $Q$ is a polynomial with $Q\left(w_{1}\right) \neq 0$ and $U$ an entire function. It follows by $\rho(H)<+\infty$ that $W$ is a polynomial. Hence there exists a polynomial $P(z)$ such that $H(z)=$ $Q\left(w_{1}+\exp W(z)\right) \cdot \exp P(z)$. And we easily get the equality $G(z)+S(z)+P(z)+q W(z)$ $=U(g(z))+2 k_{0} \pi i$ for some integer $k_{0}$. If $U(w)=$ constant, then $G(z)=$ constant. This is a contradiction. Hence $U(w) \neq$ constant. By the same method as in case (II), we can easily get the factorization of case (iii).

(IV) The case when $g$ is transcendental meromorphic and $f$ is a nonpolynomial rational function. The case can be reduced to case (III) by some suitable linear linear transformation.

Thus we complete the proof of Theorem 3 .

Proof of Corollary 2. According to Theorem 3, we only need prove that there do not exist an entire function $U(w)$ of order zero and a rational function $Q(w)$ such that

$$
\cos ^{2 k+1} z=U(g), \quad \cos ^{n} z \cdot \sin z=Q(g),
$$

where $g$ is a periodic entire function with period $2 m \pi$, for some positive integer $m$.

Otherwise, suppose that there are $U$ and $Q, g$ to satisfy (21). Evidently, $U$ is a polynomial. Let $w_{1}$ and $w_{2}\left(w_{1} \neq w_{2}\right)$ be both zeros of $U(w)$ of multiplicity $m_{1}$ and $m_{2}$, respectively, where $m_{1} \neq 2 k+1$ and $m_{2} \neq 2 k+1$ and let $z_{\imath}(i=1,2)$ be any roots of $g=w_{\imath}(i=1,2)$ of multiplicity $n_{\imath}(i=1,2)$, respectively. From (21), we have $n_{1} m_{1}=2 k+1$ and $n_{2} m_{2}=2 k+1$. Hence $n_{\imath} \geqq 3(i=1,2)$. However, by Nevanlinna's Theory, we know this is impossible. Therefore, $U(w)$ can be expressed as $\left(w-w_{1}\right)^{t} \cdot P^{2 k+1}(w)$, for a polynomial $P(w)$ and an integer $t$ $(0 \leqq t<2 k+1)$. We divide two cases of the following to be considered, separately.

(I) The case when $t=0$. Then we have immediately $\cos z=\xi P(g(z))$, where 
$\xi$ is a constant with $\xi^{2 k+1}=1$. Thus it shows from the latter of (21) that

$$
(\xi P)^{2 n}\left(1-(\xi P)^{2}\right)=Q^{2} .
$$

Put $Q_{1}=Q /(\xi P)^{n}$ and $P_{1}=\xi P$. Obviously, $Q_{1}$ is a polynomial. Hence (22) can be rewritten as $1=P_{1}{ }^{2}+Q_{1}{ }^{2}=\left(P_{1}+\imath Q_{1}\right)\left(P_{1}-i Q_{1}\right)$. It follows that $P_{1} \pm i Q_{1}$ are both polynomials and have no zeros. Then both $P_{1} \pm i Q_{1}$ are constants, i.e. $P_{1}$ and $Q_{1}$ is both constants. This is absurd.

(II) The case when $t \neq 0$. Then we can write $g(z)$ as $w_{1}+X^{s}(z)$, for some entire function $X$ and some integer $s$ satisfying $s t=2 k+1$. If follows from the former of (21) that

$$
\cos z=\xi X \cdot P\left(w_{1}+X^{s}\right)=\xi P_{2}(X),
$$

where $P_{2}(w)$ denotes $\xi w \cdot P\left(w_{1}+w^{8}\right)$. From the latter of (21) and (23), we easily obtain the equality $P_{2}{ }^{2 n}\left(1-P_{2}^{2}\right)=Q^{2}\left(w_{1}+w^{s}\right)$. By the same method as in case (I), we can show that $P_{2}$ and $Q$ are both constants. This also is impossible.

Thus we complete the proof of Corollary 2.

\section{REFERENCES}

[1] F. Gross, Prime entire functions, Trans. Amer. Math. Soc., 161 (1971), 219-233.

[2] H. URABE, Uniqueness of the factorization under composition of certain entire functions, J. Math. Kyoto Univ., 18 (1978), 95-120.

[3] H. URABE, On a factorization problem of certain entire functions and a theorem of Ostrovski, Bull. Kyoto Univ. Educ., Ser. B No 53 (1978).

[4] A. EDREI, Meromorphic functions with three radially distributed values, Trans. Amer. Math. Soc., 78 (1955), 276-293.

[5] G. PolyA, On an integral function of an integral function, J. London Math. Soc., 1 (1926), 12-15.

[6] F. GRoss, On factorization of meromorphic functions, Trans. Amer. Math. Soc., 131 (1968), 215-222.

[7] G.D. Song AND J. HuANG, On pseudo-primarity of the $n$-th power of prime functions, Kodai Math. J. 10 (1987), 42-48.

[8] F. GRoss, Factorizations of meromorphic functions, U.S. Government printing office, Washington, D.C.

Department of Mathematics

ANHUi University

HEFEI, P.R. ChINA 